\title{
Recommendations from Conference Committee
}

The EPS Conference Committee with G.H. Stafford, Rutherford Laboratory, as Chairman has been very active since its foundation. It has approved so far the programmes of nine Conferences and Summerschools organized by EPS Divisions and granted sponsorship to fourteen Conferences satisfying the criteria as laid down in February by the EPS Council (see Meetings Issue 1, Spring 1970),

\section{Europhysics Conferences}

A study group had been formed consisting of W. Buckel, Karlsruhe, J. Friedel, Orsay, and N. Kurti, Oxford, to define specific criteria applicable to Europhysics Conferences. It must be emphasized that «Europhysics Conference» is not just a convenient shorthand notation for any EPS meeting, but is reserved for conferences along the lines of the famous U.S. Gordon Conferences and satisfying the criteria proposed by the study group and accepted with a few modifications by the Conference Committee at its meeting on 25 September 1970 . The version as given below will be submitted to the Executive Committee and the Council for approval.

\section{General}

Europhysics Conferences should follow broadly the philosophy and organization of the U.S. Gordon Conferences, i.e.

The subject of each conference should be well-defined and of topical interest.

The number of participants should preferably not exceed 70 , and 100 should be regarded as a limit. The number of participants from any single country should not exceed one third of the total.
Attendance should be by invitation only (see Section, 3).

Europhysics Conferences should be held, as far as possible, in secluded places, away from the distractions of large towns, but with facilities for outdoor recreation. Holiday and sporting resorts out of season, boarding schools and colleges during vacations are obvious possibilities.

These conferences should normally last at least three full days, preferably four to five days, though intensive one-day seminars might be acceptable.

Long ( $45-60$ minute) introductory papers should be given by specially invited speakers or discussion leaders.

Ample time should be provided for informal personal exchanges and discussion in small groups. For example, there may be a morning session centred round two introductory talks, an afterdinner session with one introductory talk, while the afternoon is kept free for recreation and informal discussions.

There should be no published proceedings of the conferences, unless the participants agree otherwise.

\section{Organization}

This should be in the hands of at least two people from different countries, one of them preferably a resident in the country where the conference is held. The organizers will be jointly responsible for:

- drawing up the detailed programme

- choosing and inviting the discussion leaders and the other participants

- finance and the local arrangements.
They should submit a report to the EPS Conference Committee.

\section{The Participants}

Since the Europhysics Conferences are intended to be study conferences rather than "schools", participants should be chosen mainly from among those who are actively working in the particular field. Many of the participants will be selected by the organizers from among those who have applied to attend. For this selection to be comprehensive, it would seem necessary to advertise the dates and topics of the various Europhysics Conferences well ahead. This could be done by listing them separately in Europhysics News, in the Physics Bulletin of the IPPS, Bulletin de la Société Française de Physique, and other national or international physics periodicals, e.g. Physics Today.

As stated above, a total attendance figure of 100 is to be regarded as the maximum with about 70 as a desirable aim.

\section{Finance}

Each Europhysics Conference should be selfsupporting. Since it is expected that discussion leaders will not have to pay for their board and lodging and that in most cases their travelling expenses will also be borne by the conference, other participants will have to pay a more than nominal registration fee. A figure of between Sw. Frs. 400 and Sw. Frs. 500 comprising registration fee, board and lodging, may be regarded as a guide for a five day conference.

Since it is not possible to budget exactly, some conferences may show a slight profit, others a slight 
loss, and it would be advisable to establish a special Europhysics Conference Fund. This could be administered by EPS Headquarters in Geneva but should constitute a separate account. Any surplus from conferences and donations for the general purpose of Europhysics Conferences should be paid into that account which, in turn, could pay out subsidies if necessary and - more important - provide a financial guarantee. It is expected that organizers will try to get financial help from their own industry and government.

5. Initiation and Approval of Conferences

Most Europhysics Conferences will be proposed by the various Divisions of EPS although individual physicists may also suggest topics, especially in fields not properly covered by the Divisions. All Europhysics Conferences should be approved by the Conference Committee but this could be done by letter without having to wait for a committee meeting. A subcommittee will assist the Chairman by examining the proposals, ensuring that there is no overlap in time or duplication in subject among the Europhysics Conferences themselves or with other meetings, and by checking that the conditions laid down in Section 1 are fulfilled. This subcommittee could also keep an eye on the finances and ensure that all national societies are fully informed about Europhysics Conference activities.

The Conference Committee proposed that W. Buckel, J. Friedel and N. Kurti act as subcommittee as proposed in Section 5 above. All correspondence concerning approval and sponsorship of conferences should be addressed to:

\section{Conference Committee \\ European Physical Society \\ P.O. Box 309 \\ CH-1227 Carouge-Geneva \\ Switzerland}

or to Dr. L. Etienne, at the above address.

\section{Guide to Entries}

In this Meetings Issue of Europhysics News, details are given of all future meetings about which notification has been received up to the end of November 1970. The events are arranged in date order, with summerschools being listed separately. The meetings organized by Divisions of the EPS are printed in bold type and boxed; those sponsored by the EPS are printed in bold type. Europhysics Conferences are indicated by a star following the date.

In general, meetings are open to any applicants: where attendance is known to be restricted to invited applicants this is indicated accordingly.

The information is given in the following order:
Date
Title

Venue

Contact for information

(A) Application deadline / (P) Deadline for papers / Number of participants / By invitation only (inv.)

Language, if English is not solely used / Sponsorship / Conference fee

\section{Conferences}

\section{1}

\section{4- 5 January}

Computational Techniques as an Aid in Physical Metallurgy

Leeds, UK

Iron and Steel Institute,

4 Grosvenor Gardens, London SW 1

\section{5- 7 January}

Bth Annuial Solid State Physics Conference Manchester, UK

The Meetings Officer, IPPS, 47 Belgrave Square. London SW 1

\section{8 January}

3rd L.H. Gray Memorial Conference on Radiation Effects and the Mitotic Cycle

Manchester, UK

Alma Howard, Paterson Laboratories, Christie Hospital and Holt Radium Institute Manchester M20 9BX

P: 1.9 .70

\section{January}

Sorption Phenomena in Vacuum

Liverpool, UK

The Meetings Officer, IPPS, 47 Belgrave Square, London SW 1

P: 30.11 .70
13 January

Electron Spectrometry

London, UK

The Meetings Officer, IPPS, 47 Belgrave Square, London SW 1

P: 30.11 .70

\section{8 - 23 January}

International Symposium on Quantum Chemistry, Solid-State Physics and Quantum Biology

Sanibel Island, USA

Winter Institute, Nuclear Sciences Bldg, University of Florida, Gainasville, Fla. 32601

\section{9- 21 January}

Mexico International IEEE Conference on Systems, Networks and Computers

Oaxtepec, Moxico

M.A. Murray-Lasso, Instifuto de Ingenieria, Cludad Universitaria, Mexico 20

A: $20.10 .70 /$ P: $31.10 .70 / 400$

English, Spanish / US $\$ 40$

February

Chemical Analysis by Charged Particle Bombardment

Namur, Belgium

G. Deconninck, Laboratoire d'Analyse par Róactions Nuclóaires, Facultoss Universitaires de Namur, rue Muzet 22, 8-5000 Namur

15 - 15 February

National Symposium on Atomic Energy Tokyo, Japan

M. Masamoto, Secretary-General, Atomic Energy Society of Japan, e/o Japan Atomic Energy Research Institute, No, 1-13, 1-chome Shimbashi, Minato-ku, Tokyo

\section{5 - 17 February}

8th AINSE Plasma Physics Conference

Lucas Heights, N.S.W. Australia

E.A. Palmer, Exocutive Officer, AINSE.

Private Mail Bag, P.O.

Sutherland, N.S.W. 2232

A: $30.8 .70 / P: 30,8.70 / 100 /$ inv.

\section{7 - 18 February}

Electron Energy Analysis

London, UK

The Meetings Officer, IPPS, 47 Belgrave Square, London SW 1

P: 7.12 .70

\section{7 - 19 Februan}

International Solid State Circuits Conierence Philadelphia, USA

Meetings Information, IEEE,

345 E. 47 th St., New York, N.Y. 10017

\section{$24-27$ February}

Particle Physies

Hamburg, Federal Ropublic of Germany

G. Sohngen, DESY, Notkestieg 1, D- 2 Hamburg 52 P: $4.12 .70 /$ inv.

German

\section{February - 5 March}

22nd Pittsburgh Conference on Analytical Chemistry and Applied Spectroscopy

Cleveland, USA

J.F. Jackovitz, Westinghouse Research Laboratories, Pittsburgh, Pa. 15235

P: 1.10 .70

\section{3 March}

Particle Accolerator Conference

Chicago, Illinois, USA

L.C. Teng, National Accelerator Laboratory Box 500, Batavia, III. 60510

P: 15.11 .70 
1. 6 March

Nuclear and High-Energy Physics

Wurzburg, Federal Republic of Germany

U. Schmidt-Rohr, POB 1248, D- 69 Heidelberg

P: $4.12 .70 / 700$

German, English / DM 20.-

\section{1- 12 March}

10th Nuclear Physica University Weok

Schladming, Austria

Organizing Comnittee, Institut for Theoretische

Physik, Universitat Graz, Universitatsplatz 5, A. $8010 \mathrm{Graz}$

A: $20.12 .70 / 200 /$ inv.

\section{8- 11 March}

Physical Studies of Minor Planets

Tucson, Arizona, USA

T. Gehreis, Lunar and Planetary Laboratory, University of Arizona,

inv. Tucson, Arizona 8572

IAL

\section{8- 12 March}

Biophysical Aspects of Radiation Quality

Lucas Heights, N.S.W., Australia

IAEA, Kărntnerring 11, POB 590 , A- 1011 Vienna, Austris

P: $15.9 .70 / 150$

English, French, Spanish, Russian

\section{8 - 13 March}

High Polymor Physics, Molecula Physics Berlin, Federal Republic of Germany

D. Hahn, Abbestrasse 2-12, D. 1 Berlin 10

P: 4.12.70/250

German, English / DM 20,-

\section{0 - 12 March}

Photographic Science

Toronto, Canada

H.J. Hall, Society of Photo Science and Engineers, 10 Maguire Road, Lexington, Mass, 02173, USA

\section{5 - 17 March}

3rd Conference on Neutron Cross Sections and Technology

Knoxville, Tennessee, USA

J. A. Harvoy, Oak Ridgo National Laboratory, POB X, Oak Ridge, Tenn. 37831

P: (Abstracts) 7.12.70, (Papers) 15.3.71

US $\$ 27$

\section{5 - 20 March}

Extraterrestrial Physics, Mass Spectroscopy, High Froquency Physics

Giessen, Federal Republic of Germany

D. Kossel, clo Ernst Leitz GmbH, D- 633 Wetzlar

P: $4.12 .70 / 300$

Gorman, Engliah / DM 20,-

\section{6 - 18 March}

Electrical Safety in Hazardous Environments

London, UK

The Conference Department, IEE,

Savoy Place, London WC2R 0BL.

A: $16.3 .70 / P: 1.10,70 / 300$

f. 20

\section{March}

Ultrasonic Bubble Chambers

Dideot, UK

The Mectings Officer, IPPS, 47 Belgrave Square, London SW 1

A: $15.12 .71 / P: 1.2 .71 / 50 /$ inv.

\section{2 - 25 March}

IEEE International Convention and Exhibition

Now York, N.Y, USA

Meetings Information, IEEE,

345 E. 47th St., Naw York, N.Y. 10017

\section{$22 \cdot 26$ March}

Plasma Physic:

Bochum, Federal Republic of Germany

H. Schlueter, Ruhr-Universităt Bochum, Institut fü Experimentalphysik, D. 463 Bochum $P: 11.12 .70 / 200 /$ inv.

German / DM 10 -

\section{2 - 27 March}

Semiconductor Physics, Motal Physics, Low Temperatures, Thermodynamics, Surface Physics

Münster, Federal Republic of Germany

D. Kamke, University, D- 493 Bochum

P: $15.12 .70 / 700$

German, English / DM 20.-

\section{3 - 25 Mareh}

Electro-Optics International

Brighton, UK

G. Johns, Press Officer, Trident Publicity, 14 Queen Anne Terrace, North Hill, Plymouth PL4 BEG

P: 15.8 .70

\section{3 - 26 March}

The Moon

Newcastle-upon-Tyne, UK

S.K. Runcorn, School of Physics,

Univorsity, Newcastie-upon-Tyno NE1 7RL inv.

IAU

\section{26 March}

Negative lons

Liverpool, UK

The Meetings Officer, IPPS, 47 Belgrave Square, London SW 1

P: $2,1.71$

\section{3 - 26 March}

5th International Conforence on Fluid Sealing Reading, UK

Conference Secretary, BHRA, Cranfiold, Bedford A: February 1971 / P: $30.9 .70 / 250 /$ inv. e 30

\section{4 - 26 March}

Structure and Growth of Thin Films

Southampton, UK

The Meetings Officer, IPPS, 47 Belgrave Square, London SW 1 P: 1.2 .71

\section{9 - 31 March}

Magnetiam

Salzburg, Austria

K.H. Riewe, Deutsche Physikalische Gesellschaft, Horaoustrasso 9, D. 645 Hanau,

Fed. Rep. of Germany

$P=30,11.70 / 250$

German, English / DM 20.-

\section{9 - 31 March}

New Dovelopments in Reactor Mathematics and Applications

Idaho Falls, Idaho, USA

J.C. Haire, Idaho Nuclear Corporation, POB 1845, Idaho Falls, Id, 89401

P: $1.11 .70 / 300$

ANS members US $\$ 28$, non-members US $\$ 38$

\section{March - 2 April}

International Conforence on Space and Communications

Paris, France

Colloque International de l'Espace et la

Communication,

16 rue de Presles, F. 75 Paris $15 e$

P: 15.11 .70

FF 500 -
29 March - 2 April

Use of Radiation and Radioisotopes for Genetic Improvement of Induatrial Microorganisms

Vienna, Austria

IAEA, Karntnerring 11, РOB 590, A- 1011 Vienna P: 2.11 .70

\section{March - 2 April}

International Statistical Mechanics Conference Chicago, Illinois, USA

S.A. Rice, James Franck Institute, University of IUPAP

Chicago, 5640 Ellis Ave, Chicago, III, 60037

\section{March - 2 April}

Datafait 1971

Nottingham, UK

Conference Department, British Computer Society, 29 Portland Place, London W

\section{March - 3 April}

High Speed Physies and Electronics in Physical Experiments, Quantum Optics

Freudenstadt, Federal Republic of Germany

K. Friinz, o/o AEG-Telefunken, D. 79 Ulm

P: $18.12 .70 / 200$

German, English / DM 20.-

\section{March - 1 April}

2nd Israeli Vacuum Congress and Exhibition Tel Aviv, Israel

B. Raz, Instifute of Chemistry,

Tel Aviv University, Ramat Aviv

P: 1.12 .70

English, Hebrew

\section{March - 2 April}

European Semiconduetor Device Research Conference

Munich, Federal Republic of Germany

H. Weiss, Siemens Aktiengesellschaft, Abteilung 5, Balanstrasse 73, D- 8 Munich 80 $A=1.3 .71 / P=10.12 .70 / 800$

DM 50,-, students DM 10.-

\section{2 April}

Tectonic Implications of Petrochemistry Rending, UK

P.J. Smith, Department of Earth Sciences, The Open University, Walton, Bletchley, Berks.

P. (Abstracts) 11.1 .71

\section{6 April}

Vacuum Equipment

Sussex, UK

The Meetings Officer, IPPS, 47 Belgrave Square, London SW 1

P: 30.1 .71

5 - 7 April

1971 Cryogenics Conference and Exhibition Brighton, UK

British Cryogenics Council, 16 Belgrave Square, London SW 1

\section{5- 7 April}

Dieloctric Polarization and Conduction Theory; Instrumentation for Dielectric Studies London, UK

A.H. Price, Edward Davies Chemical Laboratories, University Colloge of Wales, Aberystwyth, Cards. A: February 197

\section{5 - 7 April}

Elementary Particle Physics

Lancaster, UK

The Meetings Officer, IPPS, 47 Belgrave Square, London SW 1 


\section{5- 7 April}

International Conference on Duality and Symmetry in Hadron Physics

Tel Aviv, Israel

Y. No'eman, Department of Physies and Astronomy, Tel Aviv University, Tel Aviv

A: $1.12 .70 / P=15.2 .71 / 130 /$ inv.

IUPAP / US $\$ 25$

\section{7 April}

Developments in Electric and Magnetic Resonance Keole, UK

E.F. Slade, Department of Physics, University of Keole,

Keele ST5 5BG, Stafiordshire

\section{5 - 8 April}

Ird National Atomic and Molecular Physics Conference

York, UK

The Meetings Officer, IPPS, 47 Belgrave Square, Londan SW 1

A: $1.3 .71 / P: 20.1 .71 / 250 /$ inv.

E. 25 (incl. accommod.

\section{5 - 8 April}

International Conference on Conduction in Low Mobility Materials

\section{Eilat, Israel}

D.S. Tannhauser, Solid State Institute, Technion, Israel Institute of Technology, Haifa A: $1.12 .70 /$ P: $5.4 .71 / 100$

\section{us $\$ 30$}

\section{6-8 April}

Jubilee Congress of the Netherlands' Physical Society

Utrecht, The Notherlands

J. Kuperus, Transitorium 1, Leuvoniaan 21, Utrecht Dutch / no feo

\section{3 - 15 April}

International Symposium on Computers and Automata

New York, N.Y., USA

Polytechnic Institute of Brooklyn, MRI Symposium Committee, 633 Jay St., Brooklyn, N.Y. 11201

\section{3- 15 April}

Fast Reactor Fuel Element Technology New Orleans, Louisiana, USA

J.A. DoMastry, Weatinghouse Astronuclear, Box 10964, Pittsburgh, $\mathrm{Pa}, 15236$

P. $15.10 .70 / 400$

\section{3- 16 April}

9th International Conference on Magnetics Denver, Colorado, USA

Meetings Information, JEEE, 345 E.47th St. New York, N.Y, 10017

\section{16 April}

Meson Resonances and Related Magnetic Phenomena

Bologna, Italy

A. Zichichi, Istituto di Fisiea "A. Righi", 200 Via Imerio 46, I- 40126 Bologna

\section{5 - 16 April}

Surface, Vacuum and Space

Liège, Belgium

Belvac, Avenue de la Renaissance 30, B. 1040 Brussels

A: $15.3 .71 / P: 1.11 .70 / 150$

Dutch, German, English, French /

IUVSTA members FB 420.-,

non-members FB $560 .-$, students FB 70.-

\section{5- 21 April}

Structure of " $1 \mathrm{fy} / 2$ "Nuclei

Legnaro, Padua, Italy

R.A. Ricci, Laboratori Nazionali di Legnaro Via Vittorio Emanuele III 4, 1-35020 Legnare. Padua

A: $15.2 .71 / P: 15.3 .71 / 80$

L. 5000

\section{8 - 21 April}

Neutron Sources and Application

Augusta, Goorgia, USA

C. Ice, Savannah River Laboratory, Aiken, S.C. 29001

P: $3.1 .71 / 300$

ANS members US $\$ 25,-$ non-members US $\$ 35$.-

\section{9- 22 April}

The Perception and Application of Flashing Lights London, UK

W.D. Wright, Physies Department,

Imperial College, Prince Consort Road, IUPAP London, SW 7

\section{$19 \cdot 23$ April}

5th Conference on Magnetohydrodynamic Electrica Power Generation

Munich, Foderal Republic of Germany

Mme J. Faure, ENEA, 38 Boulevard Suchet, F. 75 Paris 160 , France

A: $1.1 .71 / P \cdot 1.10 .70 / 400$

English, French, Russian

\section{9 - 23 April}

Radioisotopes and Radiation Effects

Anaheim, Califomia, USA

American Society for Testing and Materials. 1916 Race St., Philadolphia, Pa. 19103

\section{6 - 30 April}

International Congress on Protection against Accelerator and Space Radiation

Geneva, Switzerland

Scientific Conference Secretariat, CERN, CH. 1211 Genova 23

A: $1.2 .71 / 200 /$ inv.

English, French, German / Sw.Frs, 150,-

May

Ergonomics and Occupational Hygiene for Safety Officers in Industry

Salford, UK

The Registrar, Room 111 A, University of Salford Salford, M5 4WT

$50 /$ inv.

\section{8 May}

3rd International Conference of Space Technology Rome, Italy

G.A. Partel, Centro Studi Trasporti Missilistici, Via Squarcialupo 19 A, 1. 00162 Rome

A: 31.1 .71 / P: (Abstracts) 31.12.70, (Papers) 1.4.71 300

English, French, Italian, Russian / participants L 20000 , authors L 15000

\section{9 - 15 May}

Rotation of the Earth

Marioka, Mizusawa, Japan

P. Melchior, Observatoire Royal de Belgique, 3 av. Circulaire, Uccle,

inv. B- Brussels 18, Belgium

IAU

\section{0 - 13 May}

3rd Conference on Static Electrification London, UK

The Meotings Officer, IPPS, 47 Bolgrave Square, London SW1

P: 1.2 .71

\section{2- 14 May}

Electron, Ion and Laser Beam Technology

Boulder, Colorado, USA

Meetings Information, IEEE, 345 E,47th St. New York, N.Y, 10017

\section{19 May *}

Physics of Selenium and Tellurium

Pont-â-Mousson, France

M. Hulin, Groupe de Physique des Solides, Tour 23, 9, quai Saint-Bernard, F- 75 Paris $5 \mathrm{e}$ A: $15.4 .71 / P: 31.3 .71 / 60$

FF 450,-

\section{6 - 20 May}

International Microwave Symposium Washington, D.C., USA

R.V. Garver, Harry Diamond Laboratories, Conn, Ave. \& Van Ness St., Washington, D.C. 20438

\section{7 - 20 May}

Spring Joint Computer Conference

Atlantic City, N.J., USA

AFIPS Headquarters, 210 Summit Ave., Montvale, N.J. 97645

\section{$17-28$ May}

Practical Electron Microscopy

Salford, UK

Administrative Assistant, Room $111 \mathrm{~F}$, Univeraity of Salford, Salford M5 4WT A: $10.5 .71 / 24 /$ inv.

\section{4 - 26 May}

5th International Symposium an Photon Detectors Vama, Bulgaria

Bulgarian Academy of Sciences, Organizing Committee, 5th Int. Symp. on Photon Detectors.

72 Lenin Boulevard, Sofia 13

Q / Inv

German, English, French, Russian / US \& 10

\section{5 - 29 May}

Journées de Physique de 1971 Evian, France

Société Française de Physique,

33 rue Croulebarbe, F- 75 Patis 13 e

French / FF 20.-

\section{June}

The Eclipse of 7 March 1970

Seattle, USA

COSPAR, 55 Boulovard Malesherbes, F- 75 Paris 8e, France URSI, IAU, IAGA

June

Dynamics of the Thermosphere and Ionosphere above $120 \mathrm{~km}$

Seattle, USA

COSPAR, 55 boulevard Malesherbes. URS! F. 75 Paris 8 e, France

\section{1 - 5 June}

Two-Body Reactions at High Energies Titisee, Federal Republic of Gormany

The Secretary, Institut für theor. Kemphysik der Universitat,

Kaiserstrasse 12, D. 75 Karlsruhe

A: $26.4 .71 / 50 / \mathrm{inv}$

DM 20,-

2. 5 June

3rd International Conference on Digital Computer Applications to Process Control

Helsinki, Finland

3rd IFAC/IFIP Conference, Box 10192 Helainki 10 
7. 11 June

High Enorgy Collisions on Nuclei and Particle Productions

Titisee, Federal Republic of Germany

The Secretary, Institut fur theor.

Kernphysik der Universitlat,

Kaiserstrasse 12, D- 75 Karlsruhe 1

A: $3.5 .71 / 50 /$ inv.

DM 20.-

\section{4 - 18 June}

European Association of Radiology, 2nd Congress Amsterdam, The Nethorlands

2nd Congress of the European Association

of Radiology, cio Holland Organizing Contre,

16 Lange Voorhout, The Hague

P: 15.1 .7

14 - 18 June

Evolutionary and Physical Problems of Meleoroids Albany, Now York, USA

R.E. McCrosky, Smithsonian Astrophysical

Observatory, 60 Garden St., Cambridge, Mass. 02138

inv.

IAU

\section{4- 18 June}

26th Annual Symposium on Molecular Structure and Spectroscopy

Columbus, USA

K. Narahari Rao, Department of Physics, Molecular Spectroscopy Symposium

174 West 18 th Avenue, The Ohio State University, Columbus, Ohio 43210

\section{5- 17 June}

9th Annual Meeting of the European High Pressure Research Group

Umeả, Sweden

G. Bäckström, University of Umeả, Department of Physics, S- 90187 Umeả

A: $30.4 .71 /$ P: $30.4 .71 / 100$

English, German, French

\section{6 - 18 June}

Heat Exchangors

Paris, France

H. Perdon, IFCE, 3, rue Henri-Heine, F. 75 Paris $16 \mathrm{e}$

A: $1.6 .71 / 400 /$ inv,

French, English

\section{7 - 23 June}

4th Contarence on Plasma Physics and Controlled Thermonuclear Fusion

Madison, Wisconsin, USA

IAEA, Kärntnetring 11, РОВ 590 A. 1011 Vinnna, Austria

\section{1 - 24 June}

5th Symposium on Temperature - Its Measurement and Control in Scionce and Industry

Washington, D.C., USA

V.J. Giardina, Instrument Socioty of America, 530 William Ponn Place, Pittsburgh, Pa. 15219 P: 4.1 .71

\section{1 - 25 June}

Electromagnetic Interactions

Trieste, Italy

The Deputy Director, International Centre for Theoretical Physics POB $588,1.34100$ Trieste

\section{1 - 25 June}

Health Physics Society, 16th Annual Meoting New York, N.Y, USA

R.F. Cowing, Executive Secretary

Health Physics Socioty, 194 Pilgrim Road, Boston, Ma. 02215

\section{$21-26$ June}

Theoretical Physics and Biology

Versailles, France

M. Marois, Instifut de la Vie,

Bg Boulevard Saint Michel, F. 75 Paris $5 e$ IUPAP

27 June -2 July

8th World Energy Conference

Bucharost, Romania

Romanian National Committee of the World Energy

Conference, Bd. Lacul Tei 1, Sectoroul 2, Bucharest 30

A: $1.3 .71 / P=1.11 .70 / 3000 /$ in

English, French, Romanian / US \& 80

\section{June - 2 July}

2nd Symposium on Fluidic

Prague, Crechoslovaki

2nd Symposium on Fluidics, IFAC.

Techniska 4, Prague B

29 June - 1 July

Science and Civilization

London, UK

The Diractor, CIBA Foundation,

41 Portland Place, London W1N 4BN

30 June - 6 July

Amsterdam International Conference on Elementary Particles

Amsterdam, The Netherlands

Miss E.W.D. Steel, Conference Secretary, CERN, CH- 1211 Geneva 23, Switzerland P: 1.5.71 / 550 / inv.

Fl. 150 .

July

4th International Conference on Science and Society - Man and his Environmen

Herceg-Novi, Yugosiavia

Konforencija Nauka i Drustvo,

Organizacioni odbor, POB 163, Belgrade

A: $1.1,71 /$ inv

English, French, Russian, Yugoslav

\section{5 - 7 July}

Rare Earths and Actinides

Durham, UK

Solid State Physics Sub-Committee, IPPS

47 Belgrave Square, London SW

P: $3.4 .71 /$ inv.

\section{5 - 8 July}

8th International Shock Tube Symposium London, UK

J.L. Stollory, Symposium Sacratary. Department of Aeronautics, Imperial College.

Prince Consort Road, London SW 7

P: (Title) 1.9.70, (Manuscript) 1.1.7

\section{5 - 9 July}

Building Acoustica

Salford, UK

The Registrar, Room 111 A

University of Salford, Salford M5 4WT

A: $21.6 .70 / 30 /$ inv

\section{5 - 9 July}

3td International Conference on Crystal Growth Marsoille, France

B. Mutaftchiev, Université d'Aix-Marseille, CNRS, Saint-Járôme 13, F. 13 Marseille 13 e IUCr, IUPAC, IUPAP

\section{5 - 10 July}

7th International Conference on General Relativity and Gravitation

Copenhagen, Donmark

C. Moller, Nordita, Blegdamsvoj 17

DK- 2100 Copenhagen 6 IUPAP
6. 9 July

3rd Annual Conference of the Atomic Spectroscopy Division (EGAS)

Reading, UK

G.W. Series, J.J. Thomson Physical Laboratory. Whiteknights, Reading RG6 2AF, Berkshire

P: (Abstracts) 20.12.70, (Papers) 15.5.71 / 200 inv.

8. 11 July

Nuclear Three Body Problem

Budapest, Hungary

Eơtvōs Lorand Physical Sociely,

Szabadsag-tér 17, Budapest V

A: $1.5 .71 / P: 1.6 .71 / 150 /$ inv.

US $\& 10$

9 July

Photon-Phonon Interactions

York, UK

The Meetings Officer, IPPS, 47 Belgrave Square, London SW 1

A: $1.6 .71 / P: 1.5 .71 / 100 / \mathrm{inv}$

19 - 23 July

2nd International Conference on Light Scattering in Solid

Paris, France

M. Balkanski, Laboratoire de Physique des Solides Tour 13, Faculté des Sciences

9 quai Saint-Bernard, F- 75 Paris $5 e$

A: $15.1 .71 / P: 15.2 .71 / 200$

English, French / IUPAP / US \& 75

\section{$21 \cdot 23$ July}

Visual Performance When Using Optical Instruments Munich, Federal Republic of Germany

H.A.W. Schober, Institut for Med. Optik Universitat Munchen, Barbarastrasse 16, IUPAP D. 8 Munich 13

\section{5 - 31 July}

23rd International Congress of Pure and Applied Chemistry

Boston, Mass., USA

A.T. Winstead, Director

c/a American Chemical Society,

1155 Sixteenth Street N.W.

Washington, D.C. 20036

IUPAC

26 - 31 July

7th Intornational Conference on the Physics of Electronic and Atomic Collisions

Amsterdam, The Notherlands

J. Kistemaker, FOM-Instituut voor Atoom- on

Molecuulfysica, Kruislaan 407 , Amsterdam-Watergraafsmeer IUPAP

31 July - 13 August

Aurora and Airglow

Moscow, USSR

A. Omholt, Instituto of Physics, University of Oslo Posthops 1048, Blindern, Oslo, Norway

P. $1.3 .71 / 150$

IAGA, IAU, URSI, ICSU / US \& 2

\section{2- 5 August}

2nd Conference on Applied Physical Chomistry

Veszprem, Hungary

Organizing Comm. Physical Chemistry, Vegyipari Egyotem, Analitikai Kemia Tanszek, Veszprem

\section{8 - 13 August}

4th International Conference on Amorphous and Liquid Semiconductors

Ann Arbor, Michigan, USA

M.H Cohen, James Franck Institute, 5640 S, Ellis, Chicago, III, 6063?

P: 1.4 .71

IUPAP 


\section{9- 11 August}

Reactor Operating Experience

Denver, Colorado, USA

C.E. Branyan, APDA - 1911 First St,

Detroit, Mich. 48226

\section{August}

Laser and Radar Measurements in Cloud Physics Moscow, USSR

W.L. Godson, Meteorological Service, Department of Transport,

315 Bloor Street W.

Toronto 181, Ontario, Canada

P: 15.2 .71

English, French / LAMAP, IUCRM

13- 21 August

Wolf-Rayet and High Temperature Stars

La Plata, Argentin

M.K. Vainu Bappu, Astrophysical Observatory,$$
\text { Kodaikanal, India }
$$

\section{6 - 25 August}

12th International Conference on Cosmic Rays Hobart, Tasmania, Australia

K.B. and A.G. Fenton, University of Tasmania Department of Physics, IUPAP

Box 252 C, GPO Hobart, Tasmania 7001

\section{8 - 24 August}

13th International Congress of the History of Science

Moscow, USSR

Organizing Committee of the 13th International Congress of the History of Science, 2000 Staropansky $1 / 5$, Moscow K-12

English, French, German, Russian / US \$ 25

\section{8 - 26 August}

7 th International Congress on Acoustics Budapest, Hungary

T. Tarnoczy, Acoustical Resoarch Group Puskin u, 5-7, Budapest VIII

A. $31,10.70 / P \cdot 15,12.70 / 1400$

Engliah, French, German / IUPAP / US 835

\section{3 - 27 August}

Statiatical Properties of Complex Nuclear Systems Albany, N.Y., USA

J. B. Garg, SUNY, 1400 Washington Avenue, Albany, N.Y. 12209

IUPAP

\section{3 - 27 August}

International Federation for Information Processing, 5th Triennial Conference

Ljubljana, Yugoslavie

The British Computer Society,

29 Portland Place, London W 1, UK

\section{3 - 27 Augus!}

International Symposium on Electron and Photon Interactions at High Energies

Ithaca, N.Y., USA

B.D. McDaniel, Laboratory of Nuclear Studies, IUPAP Cornell University, Ithaca, N.Y. 14650

\section{5 - 28 August}

International Symposium on Kepler's 400th Anniversary

Leningrad, USSP

Organizing Committee of the 13th International Congress of the History of Seience. Staropansky $1 / 5$, Moscow K-12

English, French, German, Russian / US \$ 25

\section{August - 2 September}

3rd International Conference on Vacuum UV Radiation Physics

Tokyo, Japan

K. Ishiguro, Univeraity of Tokyo, College of Further Education, Komaba, Tokyo 158 IUPAP
30 August - 10 September

Advanced Study Institute on Earth's Particles and Fields

Cortina, Italy

B.M. McCormac, Lockheed Palo Alto Research Laboratory, 3251 Hanover, Palo Alto, Cal, 94304, USA

US $\$ 50$

\section{August - 2 September}

Ultraviolet and X-Ray Spectroscopy in Astrophysical and Laboratory Plasmas

Lunteren, The Netherlands

C. de Jager, Space Research Laboratory of the Astronomical Institute. Benoluxlaan 21, Utrecht

IAU

\section{August - 3 September}

New Directions and Frontiers in Variable Star Resoarch

Bamberg, Federal Republic of Germany

G.H. Herbig, Lick Observatory,

University of California,

Santa Cruz, Cal. 95060, USA

inv.

IAU

\section{August - 3 September}

10th International Symposium on Free Radicals Lyon-Villourbanno, France

M. Peyron, Département de Chimie, INSA, F. 69 Villeurbanne

\section{September}

Analytical Procedures for Eelipsing Binary Light Curvos

Philadelphia, USA

R.H. Koch, Department of Astronomy,

University of Pennsylvania, Philadelphia, Penn. 19104

IAL

\section{September}

Electric and Magnetic Properties of Dilute Alloys Eger, Hungary

L. Pal, POB 49, Budapest 114

100

Russian, English f US \$ 25

\section{September - October}

Spectral Classification and Multicolor Photometry Cordoba, Argentina

J. Landi Dessy, Observatorio Astronomico de Cordoba, Cordoba, Argentina

\section{1- 3 September}

Pattern Recognition

Brighton, UK

The Meotings Officer, IPPS, 47 Belgrave Square, London SW 1

\section{1- 8 September}

Annual Meeting of the British Association for the Advancement of Science, Plenary Session Swansea, UK

R.P. Saville, British Asaociation for the Advancement of Science, 3 Sanctuary Buildings 20 Great Smith Street, London SW 1

A: August 1971 / 2000 / inv.

\section{L}

\section{B September}

6th International Conference on Photochemistry

Bordeaux, France

J. Joussot-Dubien, Faculte des Sciences de Bordeaux,

351 Cours de la Libération, F- 33 Talence
6. 10 September

4th International Conference on Atomic Masses and Fundamental Physical Constants

Teddington, Middlesex, UK

E.R. Cohen, Science Center, North American

Rockwell Corporation, 1049 Camina Dos Rios, Thousand Oaks, Cal. 91360, USA

A: $31.5 .71 / P: 1.4 .71 / 100 /$ iny.

IUPAP / US \& 50

\section{10 September}

Colour Centren in lanic Crystals

Reading, UK

E.W.J. Mitchall, J.J. Thomson Physical Laborntory, The University, Whiteknights, Reading RG6 2AF, Berkshite

A: $1.6 .71 / P: 1,6.71$

IUPAP

\section{6 - 11 September *}

Metal-Insulator Transitions and the Development of Narrow Energy Bands

Aussois, France

A.D. Yoffe, Cavendish Laboratory,

Free School Lane, Cambridge CB2 3RQ, UK

A: February 1971 / P: February 1971 / 100

English, French / US \$ 50

G. 16 September

4th International Conference on the Peaceful Uses of Atomic Energy

Geneva, Switzorland

United Nations, Palais des Nations,

CH. 1211 Geneva 10

\section{7 - 10 September}

IEE Joint Conference on Displays

Loughborough, UK

IEE, Savoy Place, London WC2R OBL

P: 14.12 .70

\section{$7-12$ September}

International Conference on High Energy Physics and Nuclear Structure

Dubna, USSR

V.P. Dzhelepov, Laboratory of Nuclear Problems. Joint Institute for Nuclear Research,

Head Post Office Box 79 . Moscew IUPAP

\section{0 - 15 September}

Electromngnetic Wave Theory

Tbilisi, USSR

V.V. Shovchenko, Soviet National Committee of URSI, Prospekt Marksa 18, Moscow Centre GSP.3

P: 1.3.71

Engliah, Russian / URSI / US \& 25

13 - 18 September

10th International Conference on Phenomena in Ionized Gasos

Oxford, UK

R.N. Franklin, Engineoring Laboratory, PaPA

\section{3 - 18 September}

Dynamics of lonized Gases

Tokyo, Japan

Professor Sato, Institule of Space and Aeronautical Sciences, University of Tokyo, Komaba, Meguro-ku

$100 /$ inv.

IUTAM

\section{4 - 16 Septomber}

2nd International Discussion Meating of the IMEKO Sub-Committee on Mensurement of Force and Weight

Delft I The Hague, The Netherlands

Congress Bureau Inter Scientias, POB 9058, The Haguo

A: $1.8 .71 / P: 31.1 .71 / 300$

until $18871 \mathrm{Fl}, 150$ - 


\section{4 - 17 September}

Physies of Condensed Matter: Metals and Phase Transformations

Florence, Italy

G. Boato, Istituto di Fisica dell'Università, Viale Benedetto XV 5, 1- 16132 Genoa

$A: 1.2 .71 / P: 1.6 .71 / 600$

\section{17 September}

Tst European Biophysics Congress

Baden, near Vienna, Austria

Mrs. E. Weidenhaus, Wiener Medizinische Akadomie, Stadiongasse 6-8, A- 1010 Vienna IUPAB

\section{September onwards}

Hollow-Cathode Discharges and their Applications Orsay, France

J.L. Deleroix, Plasma Physics Laboratory, Faculty of Sciences, F- 91 Orsay

English, French

20 - 23 Seplember

2nd European Meeting on Ferroelectricity

Dijon, France

L. Godefroy, Laboratoire de Díblectriques Ferroélectriques, Faculté des Sciences, Boulevard Gabriel 6, F- 21 Dijon

A: 14.7.71/P: 1.6.71/200

IUCr / FF 80.-

\section{$20-24$ Septomber}

International Conference on Atomic Collisions in Solids: Physics of Channeling and Related Phenomena

Gausdal, Norway

N.G.E. Johansson, Research Institute for Physics, S. 10405 Stockholm 50, Sweden

A: $1.4 .71 /$ P: $1.6 .71 / 200$

US 835

\section{0 - 24 September}

Cosmic Plasma Physics

Frascati, Italy

K. Schindler, ESRIN, POB 64, 1- 00044 Fraseati

A: $31.3 .71 / P: 31.3 .71 / 80$

US $\$ 10$

\section{0 - 24 September}

ath International Conference on High Energy Accelerators

Goneva, Switzerland

K. Johnsen, ISR Department, CERN, $\mathrm{CH}-1211$ Geneva 23

$350 /$ inv.

IUPAP

\section{0 - 24 September}

5th Conference on Molecular Spectroscopy

Brighton, UK

C.H. Maynard, Administrative Secretary, The Institute of Petroleum,

61 New Cavendish Stroot, London W 1

\section{$20-24$ September}

International Conference on Physics of Quiescent Plasmas

Copenhagen, Denmark

V.O. Jensen, Research Establishment Risbe, DK. 4000 Roskilde

A: $25.6 .71 / 120$

US 825

\section{1 - 23 September}

International Conference on Modern Developments in Flow Measurement

Harwell, UK

F.K. Byne, AERE, Harwoll, Didcot, Berkshire

A: $2.7 .71 / P: 1.3 .71 / 150 /$ inv.

\&. 20

\section{24 September}

Nuclear and Particle Physics

Oxford, UK

The Meatings Officer, IPPS, 47 Belgrave Square London SW 1

A: $30.7 .71 / \mathrm{inv}$.

\section{September - 1 October}

3rd International Congress on Spectrometry of Absorption and Atomic Fluorescence

Paris, France

Secretary of the 3rd CISAFA, Groupement pour l'Avancement des Méthodes Spectrographiques,

1 rue Gaston-Boissier, F- 75 Paris 15 e

\section{September - 1 October}

Crystallographic Studies at High Temperatures Odeillo, France

M. Foex, Laboratoire des Ultra Réfractaires, BP5, F. 66 Odeillo

$\mathrm{P}=1.3 .71 / 80 / \mathrm{inv}$

English, French

11 - 18 Oetober

International Conference for Solid Surfaces Boston, Mass., USA

C.B. Duke, Department of Physics, University of IUPAP IIInois, Urbana, III. 6180

\section{5 November}

Nuclear Selence

San Francisco, Cal., USA

IEEE, 345 E. 47th S1., Now York, N.Y: 10017

\section{5 - 18 November}

Fall Joint Computer Conference

Las Vegas, Nevada, USA

AFIPS Headquarters, 210 Summit Avenue, Montvale, N.J. 07645

\section{6-8 December}

Ultrasonice

Miami Beach, Fla., USA

IEEE, 345 E. 47 th St., New York, N.Y, 10017

\section{5 - 16 December}

The Mechanical Properties of Ceramics, Fibres and Composites

London, UK

The Meotings Officer, IPPS, 47 Belgrave Square, London SW 1

P: 4.9 .71

\section{2}

\section{Date not yet fixed}

Fine Structure of Tropospheric Layers

Seattle, USA

B.R. Bean, ESSA, Boulder, Col. 80302

$50 /$ inv.

English, Franch / IUCRM

\section{Spring}

Semimetals and Narrow Gap Semiconductors

Bellevue, France

M. Rodot, Laboratoire de Physique des Solides, 100

\section{April}

Reactor Materials Performance

Richland, Wash., USA

J.E. Cunningham, ORNL, $P O B X$

Oak Ridge, Tenn. 37830

\section{6 - B April}

Low Temperature Physics

Freudonstadt, Federal Republic of Germany

W. Klose, Institut fur FestkBrperphysik,

University of Saarland, D- 66 Saarbrocken

\section{$10-12$ April}

Thin Films

York, UK

The Secretary. Thin Films and Surfacen Group, IPPS, 47 Belgrave Square.

17 - 19 April

Nuclear Physics

Birmingham, UK

The Administrative Officer, Department of Physics, University of Birmingham,

POB 363, Birmingham B15 2TI

$300 /$ inv.

\section{4 May}

Advanced Power Reactors

Philadelphia, Pa., USA

A.E. Swanson, Black \& Veatch,

Kansas City, Mo. 64114

\section{7 - 11 May}

International Quantum Electronics Conference

Montreal, Quobec, Canada

IEEE, 345 E. 47th St. New York, N.Y, 10017, USA

July - August

3rd International Conference on Medical Physics Gothenburg, Sweden

S. Benner, Langasliden 22, S. 41270 Gothenburg

\section{3 - 5 July}

Band Structure in Solids

Exotor, UK

E.P. Woblfarth, Department of Mathematics,

Imperial College, Exhibition Road, London SW 7

19 - 21 July

International Conference on Radiation Damage and Defects in Semiconductors

Reading, UK

J.E. Whitehouse, J.J. Thomson Physical Laboratory, Whiteknights, Reading RG6 2AF

\section{July}

11th International Conference on the Physics of Somiconductors

Warsaw, Poland

L. Sosnowski, University of Warsaw,

Krakowskie Przedmiescie 26-28, Warsaw

August

17th URSI General Assembly

Warsaw, Poland

URSI, 7 Place Emile Danco, URS B. 1180 Brussels, Belgium

\section{11 August}

3rd International Conference on Atomic Physics.

Boulder, Colorado, USA

S.J. Smith, JILA, University of Calarado,

Boulder, Col. 80302

300 linv.

\section{18 August}

Physics and Chemistry of lce

Ottawa, Canada

M.K. Ward, Executive Secretary, International Symposium on the Physics and Chemistry of Ice, c/o National Research Council of Canada. Montreal Road, Ottawa 7

\section{1 - 25 August}

Extended Atmospheros and Circumstallar Matter in Spoctroscopic Binary Systems

Victoria, B.C., Canada

K.O. Wright, Dominion Astrophysical Observatory, Victoria, B.C.

inv

IAU

\section{August - 7 September}

9th Goneral Assembly and International Congress of Crystallography

Kyoto, Japan

Y. Saito, Executive Secretary, Japanese Organizing Committee, 9th IUCr Congress, The Institute for Solid State Physics, The University of Tokyo, 


\section{September}

Molecular Spectroscopy

Wroclaw, Poland

Institute of Low Temperatures and Structura Research, Polish Academy of Sciences,

PI. Katedralny 1 , Wroclaw

150 / inv.

English, German, Russian, French / US \& 15

\section{5- 12 September}

5th European Congress on Electron Microscopy (EMCON 72)

Manchester, UK

L. Lawrence, Administrative Secretary, EMCON 72, IPPS, 47 Belgravo Squaro, London SW 1

\section{5 - 12 Soptember}

Nuclear Structure Study with Neutrons

Balatonfured, Hungary

D. Kiss, Executive Secretary of the Organizing Committee, Contral Rusearch Institute of

Physics, POB 49, Budapest 114

300

English, Russian / IUPAP

\section{EPS General Conference}

\section{7 October}

Trends in the Development of Modern Physics

Wiesbaden, Federal Republic of Germany

European Physical Society, POB 300 CH- 1227 Carouge-Geneva, Switzerland

\section{Summerschools}

\section{1}

\author{
20 January - 12 March \\ Nucleat Theory Course \\ Trieste, Italy \\ The Deputy Director, International Centre for \\ Theorotical Physics, POB 586, 1. 34100 Trieste \\ A: $15.11 .70 /$ inv.
}

\section{26. February}

Reactor Engineering Induction Course Salford, UK

R.S. Tebble, Department of Pure and Applied Physics, University of Salford, Salford M5 4WT $10 /$ inv.

\& 70

\section{5 - 27 February}

8th Annual Wintorschool of Theoretical Physics: Recent Developments in Relativistic Quantum Field Theory and its Applications Karpacz, Poland

J. Lukierski, Institute of Theoretical Physics University of Wroclaw,

Cybulskiego 36, Wroclaw

A: $30.11 .70 / 80 /$ inv.

English, Russian / US $\$ .6$

24 February - 25 March

5th Annual Accelerator Health Physics Training Course

Berkeley, California, USA

E.J. Vallario, Division of Operational Safoty,

U.S. Atomic Energy Commission,

Washington, D.C. 20545

$15 /$ inv.

\section{Febraary - 13 March}

10th Winterschool on Thooretical Elementary Particles: Concepts of Hadron Physics

Schladming, Austria

Organizing Committee, Institut ful Theoretische Physik, Universitat Graz,

Universitatsplatz 5, A- 8010 Graz

A: $15.1 .71 / 150 /$ inv

\section{2- 23 March}

Course in Space Experiment Technology Brótigny, France

Centre National d'Etudes Spatiales, Division des Relations Universitaires,

129 rue de I'Université, F. 75 Paris 7e

A: $1.12 .70 / 35$

French

\section{March - 7 April}

CERN-SIN Spring School in Intermediate Energy Physics

Zuoz, Switzerland

F. Scheck, SIN, Hochstrasse 60, $\mathrm{CH} \cdot 8044$ Zurich

\section{June}

Summerschool on Low Temperature Physics Grenoble, France

B. Dreyfus, University of Grenoble, Codex 166, F. 38 Grenoble

\section{June -2 July}

Reactor Engineering Induction Course

Salford, UK

R.S. Tebble, Department of Pure and Applied Physics, University of Salford, Salford M5 4WT

\section{26 June}

JINR School of High Energy Physics Varna, Bulgaria

1. Poykov, Bulgarian Academy of Sciences; ul. 7 Noemvri 1, Sofia

\section{June - 2 July}

International Summerschool on Crystal Growth

Leiden, The Notherland

P. Hartman, Geologisch en Minoralogisch Instituut der Rijksuniversiteit, Garenmarkt 1 B, Leiden

A: $1.2 .71 / 80$ / inv.

$\mathrm{IUCr} / \mathrm{FI} .300$.-

July

Interaction of Energetic Charged Particles with Solids

Ankara, Turkey

E. Inonu, Middle East Technical University, Ankara

At 1.6.71/P: invited only/ $80 /$ in

\section{July - 28 August}

Particle Physics

Les Houches, France

Ecole d'Eté de Physique Théorique,

F. 74 Les Houche

A: $15.3 .71 / 35 / \mathrm{inv}$

French, English / FF 1200

\section{5 - 17 July}

Material Properties of Solids

Varenna, Italy

Società Italiana di Fisica,

Via L degli Andalo 2,

1. 40124 Bologna

19 - 31 July

Developments and Boundaries of Nuclear Physics Varenna, Italy

Societs Italiana di Fisica, Via L. degli Andalo 2. 1. 40124 Bologna
26 July - 3 September

Statistical Mechanics and Associated Mathematical Problems

Washington, USA

L. Bonnefond, Conferences Coordination, Battello Seattlo Research Center.

\section{2- 14 August}

Developments in High Energy Physics

Varenna, italy

Sociota Italiana di Fisica.

Via L. degli Andalo 2

1. 40124 Bologna

\section{2 - 20 August}

Seminar Course on Computing as a Language of Physics

Trieste, Italy

The Deputy Director, International Centre for Theoretical Physics, POB 586, 1. 34100 Trieste A: $15.2 .71 /$ inv.

\section{5 - 10 September}

Summerschool on Surface Physics

York, UK

M. Prutton, Physics Department, University of York, Heslington, York YO1 SDD

A: $1.6 .71 / 30 /$ inv.

if 35

\section{6- 15 September}

International Summersehool on the Investigation of the Surface Structure of Solids by LEED and Supplementary Methods

Smolenice, Czechoslovaki

M. Laznicka, Czechoslovak Acadamy of Sciences, Institute of Solid State Physics.

Cukrovarnicka 10, Prague 6

IUCr / US \& bo

\section{$17-27$ Oetober}

Investigations of Dynamic Processes in Molecular Systems

Binz / Rügen, German Democtatic Republic

Physikalische Gesellschaft der DDR, Am Kupfergraben 7, DDR. 108 Berlin

A: $31.12 .70 /$ inr.

\section{2}

July

Plasma Physics

Les Houches, France

J.L. Delcroix, Plasma Physics Laboratory,

Faculty of Sciences, F- 91 Orsay

French, English / FF 100,-

Published by the European Physical Sociaty

Correspondence to: EUROPHYSICS NEWS European Physical Society P.O. BOX 30 $\mathrm{CH}-1227$ Carouge Geneva Switzerland

Editorial Advisory Panels G.-J, Bénó, L. Jansen, E.N. Shaw, L. Etienne. Amberg

Editor: W.S. Newman

Printed by: Ed. Cherix \& Filanose S.A. $\mathrm{CH}-1260$ Nyon, Switzerland 